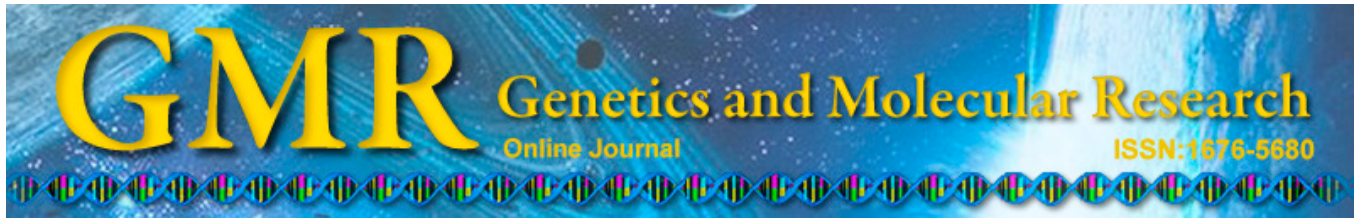

\title{
Hemodynamic changes of unexplained syncope patients in head-up tilt test
}

\author{
X. Li ${ }^{1}$, L.L. Liu ${ }^{1}$, Y.J. Wan ${ }^{2}$ and R. Peng ${ }^{3}$ \\ ${ }^{1}$ Department of Neurology, Binzhou People's Hospital, Binzhou, China \\ ${ }^{2}$ Department of Gynecology and Obstetrics, Binzhou People's Hospital, \\ Binzhou, China \\ ${ }^{3}$ Department of Pharmacy, \\ Affiliated Hospital of Inner Mongolia Medical University, Hohhot, China \\ Corresponding author: $\mathrm{X}$. Li \\ E-mail: lixiadn@126.com
}

Genet. Mol. Res. 14 (1): 626-633 (2015)

Received August 5, 2014

Accepted November 11, 2014

Published January 30, 2015

DOI http://dx.doi.org/10.4238/2015.January.30.4

\begin{abstract}
The purpose of this study was to observe the hemodynamic changes of unexplained syncope patients in the head-up tilt test and their correlations with age and gender. Eighty-six patients with unexplained syncope were administered the basic head-up test and nitroglycerin provocation test with continuous monitoring and recording of electrocardiogram and blood pressure changes. Basic characteristics of the patients and their hemodynamic responses throughout the tests were analyzed. All 86 patients tolerated and completed the head-up test. Forty-nine $(56.98 \%)$ of the patients displayed a positive reaction, $37(43.02 \%)$ patients displayed a negative reaction. Patients were divided into groups as follows: Group A, age $\leq 35$ years; Group B, age 36-45 years; and Group $C$, age $\geq 46$ years. Older patients were more prone to chronotropic incompetence, and younger patients were more prone to an excessive increase in heart rate. Older age correlated with the occurrence of autonomic nerve reaction disorder and mixed vasovagal syncope, whereas younger age was related to the occurrence of vasodepressor type vasovagal syncope $(\mathrm{P}<0.01)$. Gender did not
\end{abstract}


significantly correlate with negative or positive head-up test results (P $=0.184$ ). During the head-up test, younger patients mainly manifested an excessive heart rate increase, whereas older patients did not have significant heart rate changes. Analyzing the hemodynamic changes in the head-up test and studying the relationships between age, gender, and hemodynamic responses are crucial to determine etiologies of syncope and select appropriate treatment.

Key words: Vasovagal syncope; Tilt test; Hemodynamic change

\section{INTRODUCTION}

Syncope is a transient loss of consciousness due to a temporary reduction of cerebral blood flow that occurs unexpectedly, accompanied by a decrease or disappearance of muscle tension, and that can spontaneously recover after a few seconds to minutes. Syncope can be induced by cardiovascular diseases, neurological diseases, and metabolic diseases. However, on the basis of clinical history, physical examination, and laboratory examination, many patients cannot be definitively diagnosed; thus, syncope has long been known as "unexplained syncope." With the development of medical technology, vasovagal syncope (VVS) has been identified as the main cause of unexplained syncope (Jardine, 2013; Nwazue and Raj, 2013; Raj, 2013).

VVS is a syndrome, without nerve localization signs, manifested as arterial hypotension accompanied by transient loss of consciousness that recovers spontaneously. VVS occurs when the vagus nerve is stimulated, resulting in sudden visceral, muscular, and peripheral blood vessel dilatation and bradycardia, which leads to decreased venous blood return to the heart and the subsequent reflex actions of accelerated heart rate and increased cardiac contraction. In some cases, overexcitation of the vagus and parasympathetic nerves may cause sudden heart rate deceleration and peripheral vascular dilation, resulting in hypotension and brain hypoxia (Sheldon et al., 2012; Skerk et al., 2012).

VVS accounts for approximately $40 \%$ of all syncopal episodes. The head-up test is a safe and effective method for diagnosing VVS. In recent years, researchers have primarily focused on the hemodynamic changes that occur during the head-up test. To enrich the relevant data, we performed the head-up test on 86 patients at our hospital from June 2012 through December 2012, aiming to explore the hemodynamic changes of VVS and their correlations with age and gender.

\section{MATERIAL AND METHODS}

\section{Study subjects}

Eighty-six unexplained syncope patients who were treated at our hospital from June 2012 through December 2012 were selected randomly for the study. Among them there were 41 male and 45 female patients, aged $20-70$ years, with a mean age of $44.6 \pm 16.2$ years. The duration of time since the onset of syncopal episodes ranged from 2 weeks to 3 years, including a total of 1-10 syncopal episodes per patient (mean $5.5 \pm 4.9$ ). No organic cardiopathies or other reasons for syncope were found through history and physical examination or various investigations based on clinical indications, including routine electrocardiogram, dynamic electrocardiogram, chest X-ray, ultrasonic echocardiography, stress test, electrophysiological examination, cerebrovascular ultrasound, and electroencephalogram. 


\section{Head-up test}

An electric head-up table (pedal type), automatic blood pressure gauge, heart rate monitor, and electrocardiograph were used for the test procedure, with defibrillator and rescue drugs available on standby. Medications that affect the autonomic nervous system were withheld from all patients 2 days before the test, and patients fasted for more than $6 \mathrm{~h}$ before the test. All tests were performed between 12:30 and 4:30 pm. Each subject lay supine on the head-up table in a resting state for $5 \mathrm{~min}$ while blood pressure and ECG monitoring devices were connected. Then, $30 \mathrm{~s}$ after blood pressure and heart rate were routinely measured, the head of the table was raised to $60^{\circ}$ for $30 \mathrm{~min}$, during which time hemodynamic parameters were measured once every 5 min. According to a previously established protocol, if the test results were positive, the patient was returned to the supine position and the test was terminated (Negrusz-Kawecka et al., 2012). If the basic head-up test was negative, the patient was treated with $0.25 \mathrm{mg}$ sublingual nitroglycerin, and then the head of the table was again raised to $60^{\circ}$ for $30 \mathrm{~min}$, during which time monitoring and conditions for test termination were the same as described above.

\section{Positive and negative test criteria}

Positive criteria: In the head-up table test with standardized operation, if a patient experienced a blood pressure decrease (systolic blood pressure $\leq 80 \mathrm{mmHg}$ and/or diastolic blood pressure $\leq 50 \mathrm{mmHg}$ or mean arterial pressure decrease $\geq 25 \%$ ) and/or heart rate decrease (sinus bradycardia $<50 \mathrm{bpm}$, sinus arrest replaced by junctional escape rhythm, transient seconddegree or higher atrioventricular block, or cardiac arrest for $>3 \mathrm{~s}$ ) accompanied by syncope or near syncope (evidenced by facial pallor, diaphoresis, chest tightness, and/or hyperventilation followed by amaurosis, dysacusis, and slow response but without loss of consciousness), the head-up test was determined as positive. Patients who did not meet blood pressure criteria but developed syncope or near syncope were judged as positive, whereas patients who had a decrease in blood pressure and/or heart rate without syncope or near syncope were judged as negative. Syncopal episodes with significantly reduced heart rate and blood pressure were classified as mixed type; those with prominent bradycardia, as cardioinhibitory type; and those with slightly decelerated heart rate, as vasodepressor type. According to the Vasovagal Syncope International Study (VASIS), two other atypical heart rate responses can also occur: chronotropic incompetence, in which the heart rate does not increase significantly (supine position to headup position at $60^{\circ}$, increased heart rate $<10 \%$ ); and excessive increase of heart rate, in which a presyncopal excessive increase of heart rate (>120 bpm) occurs (Klein et al., 2012).

Negative criteria: Patients who exhibited the following hemodynamic changes were considered to have a negative test: 1) normal response: at the start of the head-up maneuver, heart rate and diastolic blood pressure increased, but systolic blood pressure remained unchanged; 2) postural orthostatic tachycardia syndrome (POTS): heart rate increased by more than $30 \mathrm{bpm}$ or remained at $120 \mathrm{bpm}$, while blood pressure did not obviously decrease (decrease was $<20 / 10 \mathrm{mmHg}$ ); 3) autonomic nerve reactive disorder: systolic and diastolic blood pressures decreased instantly and continuously without obvious heart rate rise; 4) orthostatic hypotension: systolic blood pressure decreased by $>20 \mathrm{mmHg}$ or diastolic blood pressure decreased by more than $>10 \mathrm{mmHg}$, and heart rate increased.

\section{Statistical analysis}

All data are reported as means $\pm \mathrm{SD}$. The measurement data were analyzed by the 
$t$-test, and the numeration data were analyzed by the chi-square test. The data were subjected to multivariate analysis by logistic regression. $\mathrm{P}<0.05$ was considered to be statistically significant. All data were analyzed using SPSS 12.0.

\section{RESULTS}

\section{General information and test results}

Eighty-six patients with unexplained syncope were subjected to the head-up tilt test, and all tolerated and completed the test. Two patients only underwent the basic head up-test (head up by $60^{\circ}$ for $30 \mathrm{~min}$ ) because of heart rate and/or blood pressure issues. Forty-nine patients displayed positive responses with a positive rate of $56.98 \%$; all recovered when they were set back to the supine position. General information regarding the patients and the headup test results is shown in Table 1. Cardiac rhythms seen in patients who had positive reactions to the head-up test are listed in Table 2.

\begin{tabular}{lc} 
Table 1. General information and head-up test results. \\
\hline General information & Data \\
\hline Male/female & $41 / 45$ \\
Average age & $44.6 \pm 16.2$ \\
Age of positive patient & $41.3 \pm 15.6$ \\
Age of negative patient & $46.8 \pm 16.8$ \\
Positive (total) & $49(56.98 \%)$ \\
Positive in basic test & $8(9.30 \%)$ \\
Positive in nitroglycerin provocation & $41(47.67 \%)$ \\
Mixed VVS & $12(13.95 \%)$ \\
Vasodepressor VVS & $31(36.05 \%)$ \\
Cardioinhibitory VVS & $6(6.98 \%)$ \\
Average positive response time (min) & $30.8 \pm 5.6$ \\
Basic stage (min) & $16.8 \pm 6.4$ \\
Nitroglycerin provocation stage (min) & $10.7 \pm 5.2$ \\
Blood pressure change (mmHg) & \\
Basic systolic/diastolic pressure (average) & $106.7 \pm 9.5 / 75.8 \pm 8.6$ \\
Positive systolic/diastolic pressure (average) & $74.7 \pm 10.7 / 43.8 \pm 9.0$ \\
Heart rate change (bpm) & \\
Basic heart rate (average) & $87.4 \pm 11.7$ \\
Positive heart rate (average) & $57.5 \pm 12.6$ \\
\hline
\end{tabular}

VVS = vasovagal syncope

Table 2. Cardiac rhythm status in patients with positive results $(\mathrm{N}=49)$.

\begin{tabular}{lc}
\hline Cardiac rhythm & Case No. \\
\hline Sinus rhythm & $36(73.47 \%)$ \\
Junctional escape rhythm & $6(12.24 \%)$ \\
First-degree atrioventricular block & $2(4.08 \%)$ \\
Second-degree atrioventricular block & $1(2.04 \%)$ \\
Sinus arrest & $2(4.08 \%)$ \\
Frequent premature ventricular contraction, coupled rhythm & $1(2.04 \%)$ \\
Paroxysmal atrial flutter & $1(2.04 \%)$ \\
\hline
\end{tabular}

\section{Hemodynamic changes during the head-up test}

The types of positive reactions that occurred in patients during the head-up test were 
as follows: 1) mixed VVS: 12 cases (5 males and 7 females, 24.49\%); 2) vasodepressor VVS: 31 cases (15 males and 16 females, 63.27\%); 3) cardioinhibitory VVS: 6 cases (3 males and 3 females, $12.24 \%$ ). The types of negative reactions that occurred in patients during the headup test were as follows: 1) normal reaction: 31 cases (15 males and 16 females, 83.78\%); 2) POTS: 2 cases ( 1 male and 1 female, 5.41\%); 3 ) autonomic nerve reactive disorder: 3 cases (2 males and 1 female, $8.11 \%)$; 4) orthostatic hypotension: 1 case ( 0 male and 1 female, $2.70 \%$ ).

\section{Relationship between age and hemodynamics during the head-up test}

Patients were divided into the following 3 groups: Group A, $\leq 35$ years old; Group B, age 36-45 years; Group C, age $\geq 46$ years. The basic clinical features of the different age groups, along with 2 atypical heart rate responses and 6 hemodynamic changes during the head-up test, are shown in Table 3.

Table 3. Basic clinical features and atypical heart rate responses of different age groups.

\begin{tabular}{|c|c|c|c|c|c|}
\hline Group & Total & $\mathrm{A}(\leq 35)$ & B (36-45) & $\mathrm{C}(\geq 46)$ & $\mathrm{P}$ \\
\hline Case No. & 86 & 28 & 34 & 24 & \\
\hline Female & 45 & $14(50.00 \%)$ & $18(52.94 \%)$ & $13(54.17 \%)$ & 0.247 \\
\hline Basic head-up test (heart rate/blood pressure) & 5 & $1(3.57 \%)$ & $2(5.88 \%)$ & $2(8.33 \%)$ & 0.175 \\
\hline Nitroglycerin provocation & 81 & $27(33.33 \%)$ & $29(35.80 \%)$ & $25(30.86 \%)$ & 0.648 \\
\hline Chronotropic incompetence & 9 & $1(11.11 \%)$ & $2(22.22 \%)$ & $6(66.67 \%)$ & 0.006 \\
\hline Extensive heart rate increase & 8 & $2(25.00 \%)$ & $5(62.5 \%)$ & $1(12.5 \%)$ & 0.013 \\
\hline Negative head-up test & 37 & $11(29.73 \%)$ & $16(43.24 \%)$ & $10(27.03 \%)$ & 0.107 \\
\hline Normal response & 31 & $10(32.26 \%)$ & $12(38.71 \%)$ & $9(29.03 \%)$ & 0.319 \\
\hline Postural orthostatic tachycardia syndrome & 5 & $1(20.00 \%)$ & $2(40.00 \%)$ & $2(40.00 \%)$ & 0.368 \\
\hline Autonomic nerve reactive disorder & 7 & $4(57.14 \%)$ & $2(28.57 \%)$ & $1(14.29 \%)$ & 0.029 \\
\hline Positive head-up test & 49 & $18(36.73 \%)$ & $15(30.61 \%)$ & $16(32.65 \%)$ & 0.479 \\
\hline Positive in basic test & 8 & $3(37.50 \%)$ & $3(37.50 \%)$ & $2(25.00 \%)$ & 0.241 \\
\hline Mixed type & 12 & $3(25.00 \%)$ & $5(41.67 \%)$ & $4(33.33 \%)$ & 0.217 \\
\hline Cardioinhibitory type & 6 & $3(50.00 \%)$ & $2(33.33 \%)$ & $1(16.67 \%)$ & 0.061 \\
\hline Vasodepressor type & 31 & $16(51.61 \%)$ & $6(19.35 \%)$ & $9(29.03 \%)$ & 0.011 \\
\hline
\end{tabular}

$\chi^{2}$ contingency table test was used.

\section{Factors predicting hemodynamic changes}

By means of logistic regression, we found that age was the main predictive factor in atypical heart rate responses in the early head-up test. The occurrence of chronotropic incompetence increased with age, whereas an excessive increase of heart rate was more common in younger patients.

Older age correlated with the occurrence of autonomic nervous dysfunction and mixed VVS, while younger age correlated with the occurrence of vasodepressor VVS (Table 4).

\begin{tabular}{|c|c|c|c|}
\hline Hemodynamic change & Age $36-45$ years $(\mathrm{N}=27) \mathrm{OR}(\mathrm{CI})^{*}$ & Age $\geq 46$ years $(\mathrm{N}=30) \mathrm{OR}(\mathrm{CI})^{*}$ & $P$ \\
\hline Normal response & $0.629(0.247-1.143)$ & $1.265(0.498-2.424)$ & NS \\
\hline Postural orthostatic tachycardia syndrome & $1.275(0.253-1.745)$ & $1.026(0.483-1.658)$ & NS \\
\hline Autonomic nerve reactive disorder & $1.104(0.962-1.284)$ & $1.361(1.163-1.528)$ & $<0.05$ \\
\hline Mixed type & $0.628(0.245-1.356)$ & $0.318(0.096-0.976)$ & $<0.01$ \\
\hline Vasodepressor type & $4.529(1.693-8.047)$ & $3.749(1.267-10.65)$ & $<0.01$ \\
\hline Cardioinhibitory type & $0.429(0.059-2.539)$ & $1.022(0.859-1.362)$ & NS \\
\hline
\end{tabular}

*Compared to the age $\leq 35$-year group. $\mathrm{OR}=$ odds ratio; $\mathrm{CI}=95 \%$ confidence interval; $\mathrm{NS}=$ no statistical significance. 


\section{Relationship between gender and hemodynamic changes during the head-up test}

The average age of male patients was $43.15 \pm 13.37$ years, and that of female patients was $45.86 \pm 14.75$ years, between which the difference was not statistically significant $(\mathrm{P}=$ 0.259). No significant difference was found in the influence of gender between the negative and positive results of the head-up test $(\mathrm{P}=0.184)$. In positive cases, female patients were more prone to cardioinhibitory VVS and vasodepressor VVS $(\mathrm{P}=0.038$ vs $\mathrm{P}=0.041$, respectively). In negative cases, the difference in the hemodynamic changes between male and female patients was not statistically significant (respective results: normal response $15 \mathrm{vs} 16$, $\mathrm{P}=0.648$; POTS 1 vs $1, \mathrm{P}=0.967$; autonomic nerve reactive disorder 2 vs $1, \mathrm{P}=0.621$ ).

\section{DISCUSSION}

This study of the hemodynamic responses in unexplained syncope patients during the head-up tilt test provides useful information for individualizing treatment of VVS. In patients with severe bradycardia, i.e., cardioinhibitory VVS, pacemaker therapy is the main treatment. For those with tachycardia, negative chronotropic drugs, particularly $\beta$-receptor blockers, can be effective. Antidiuretic medications, such as fludrocortisone and angiotonics, may be useful for patients in whom hypotension is the main reaction (Benditt et al., 2012).

Normally, when the heart rate increases by $10-15 \mathrm{bpm}$, diastolic blood pressure increases by $10 \mathrm{mmHg}$ and systolic blood pressure remains unchanged. Currently, it is believed that when a normal person changes from the supine to the head-up position suddenly, the returned blood volume is reduced, resulting in increased excitability of the sympathetic nerves, reducing excitability of the vagus nerve, and resulting in heart rate increase and peripheral vasoconstriction (Dupliakov et al., 2012a,b). Abnormal responses occur as follows: 1) Autonomic nerve reactive disorder: The diastolic and systolic blood pressure are reduced instantly and continuously without obvious heart rate increase, causing loss of consciousness, accompanied by autonomic nerve dysfunctions such as hyperhidrosis, constipation, and heat intolerance. In older people, the reduction of venous blood volume return that suddenly occurs cannot generate compensatory strengthening of sympathetic nerve excitability; therefore, with the extension of head-up time, the deposited blood volume in the lower body increases, and blood pressure is progressively reduced. 2) Vasovagal syncope: This generally manifests as a sudden reduction of blood pressure accompanied by symptoms, which often occurs more than 10 min after the head-up test begins, generally accompanied by bradycardia. It is thought that at the orthostatic position of VVS patients, the returned blood volume is reduced suddenly, strengthening contractibility of the heart chambers, activating mechanoreceptors (generally stimulated by myocardial diastole), weakening output of sympathetic nerves, and strengthening activity of the vagus nerve. This causes angiectasis, heart rate reduction, cerebral hypoperfusion, and loss of consciousness. 3) Orthostatic hypotension: Blood pressure decreases by $\geq 20 \mathrm{mmHg}$ systolic or $\geq 10 \mathrm{mmHg}$ diastolic. This phenomenon is common in adolescents and elderly people or patients taking drugs, such as antihypertensive medications; 4) POTS: Within 10 min of beginning the orthostatic position, the heart rate increases by $>30 \mathrm{bpm}$ or the pulse rate increases to $120 \mathrm{bpm}$. The mechanism of POTS includes autonomic neuropathy, abnormal adrenergic receptor response, and brainstem dysregulation (Corcoran and Lambert, 2012; Kollar et al., 2012; Nigro et al., 2012).

The observation and analysis of hemodynamic changes of patients in different age 
groups during the head-up test revealed that age correlated with hemodynamic changes, and with increasing age (from Groups A to C), age-related hemodynamic changes showed a gradient distribution (Tables 3 and 4). Moreover, the changes in the middle-aged group (Group B) were closer to those seen in the older-age group (Group C). In the early test, chronotropic incompetence was most often seen in older patients, whereas excessive increase of heart rate was mostly found in young patients. The observation of chronotropic incompetence in head-up test positive patients can help to determine the need for pacemaker installation. Furthermore, head-up test negative patients had several hemodynamic changes other than normal reactions. Observation and identification of such changes is conducive to diagnosis and symptomatic treatment of other types of syncope.

This study found that an excessive increase of heart rate in the early head-up test and a vasodepressor response as positive reactions were more common in young patients. This distribution of excessive increase of heart rate in younger patients concurs with the international literature (Raj and Freeman, 2012). However, in young patients, a severe cardiac inhibitory response is more common than a vasodepressor response. The reasons for the differences in our study could be due to racial differences or bias produced by the relatively small number of cases. Chronotropic incompetence in the early head-up test is more common in older patients, and the incidence increases with age (Graff et al., 2011; Liang et al., 2012; Yao et al., 2012). This was reflected in the results of the exercise stress test. When older patients were administered the head-up test under stress, they did not have compensatory increases in heart rate, and in the case of other cardiac stressors, their heart rates also remained unchanged. Possible reasons for this are as follows: 1) cardiac aging; 2) autonomic nervous dysfunction. It is unclear which of the two etiologies is dominant, and both may be equally important. In patients who tested negative, the occurrence of an autonomic nerve reactive disorder was also related to age. Older patients are more likely to have autonomic nervous dysfunction, which also supports the above view (Aydin et al., 2012; Bae et al., 2012; Buodo et al., 2012).

The head-up test results in our study show that in patients who tested positive, vasodepressor and cardioinhibitory responses were more common in females. Based on analysis of the data, it appears that three subtypes of VVS may exist, each with different pathophysiological mechanisms involving the autonomic nerves, neurohormones, and other relevant factors. However, information is limited in this regard, so further research is undoubtedly necessary.

\section{REFERENCES}

Aydin MA, Mortensen K, Salukhe TV, Wilke I, et al. (2012). A standardized education protocol significantly reduces traumatic injuries and syncope recurrence: an observational study in 316 patients with vasovagal syncope. Europace 14: 410-415.

Bae MH, Kang JK, Kim NY, Choi WS, et al. (2012). Clinical characteristics of defecation and micturition syncope compared with common vasovagal syncope. Pacing Clin. Electrophysiol. 35: 341-347.

Benditt DG, Detloff BL, Adkisson WO, Lu F, et al. (2012). Age-dependence of relative change in circulating epinephrine and norepinephrine concentrations during tilt-induced vasovagal syncope. Heart Rhythm 9: 1847-1852.

Buodo G, Sarlo M, Poli S, Giada F, et al. (2012). Emotional anticipation rather than processing is altered in patients with vasovagal syncope. Clin. Neurophysiol. 123: 1319-1327.

Corcoran SJ and Lambert EA (2012). Vasovagal syncope - the electricity, the pump or the input pressure? J. Physiol. 590: $1775-1776$.

Dupliakov DV, Golovina GA, Gar'kina SV and Liukshina NG (2012a). Vasovagal syncope or epilepsy: how to avoid errors in diagnosis. Kardiologiia 52: 74-81.

Dupliakov DV, Golovina GA, Sysuenkova EV and Glukhova VL (2012b). Assessment of vasovagal origin of syncope 
based on history features. Kardiologiia 52: 55-60.

Graff B, Graff G, Koźluk E, Tokarczyk M, et al. (2011). Electrophysiological features in patients with sinus node dysfunction and vasovagal syncope. Arch. Med. Sci. 7: 963-970.

Jardine DL (2013). Vasovagal syncope: new physiologic insights. Cardiol. Clin. 31: 75-87.

Klein KM, Xu SS, Lawrence K, Fischer A, et al. (2012). Evidence for genetic factors in vasovagal syncope: a twin-family study. Neurology 79: 561-565.

Kollar B, Mikes P, Siarnik P, Klobucnikova K, et al. (2012). Vasovagal (neurocardiogenic) syncope in the clinical practice. Neuro. Endocrinol. Lett. 33: 60-66.

Liang Z, Jiayou Z, Zonggui W and Dening L (2012). Selective atrial vagal denervation guided by evoked vagal reflex to treat refractory vasovagal syncope. Pacing Clin. Electrophysiol. 35: 214-218.

Negrusz-Kawecka M, Bańkowski T, Tabin M, Paprocka M, et al. (2012). Familial predisposition to vasovagal syncope. Acta Cardiol. 67: 279-284.

Nigro G, Russo V, Rago A, Iovino M, et al. (2012). The main determinant of hypotension in nitroglycerine tilt-induced vasovagal syncope. Pacing Clin. Electrophysiol. 35: 739-748.

Nwazue VC and Raj SR (2013). Confounders of vasovagal syncope: postural tachycardia syndrome. Cardiol. Clin. 31: 101-109.

Raj SR (2013). Highlights in clinical autonomic neurosciences: non-pharmacological therapy for vasovagal syncope. Auton. Neurosci. 173: 3-5.

Raj SR and Freeman R (2012). Highlights in clinical autonomic neurosciences: vasovagal syncope - insights on diagnosis, pathophysiology and treatment. Auton. Neurosci. 168: 1-3.

Sheldon RS, Morillo CA, Klingenheben T, Krahn AD, et al. (2012). Age-dependent effect of $\beta$-blockers in preventing vasovagal syncope. Circ. Arrhythm. Electrophysiol. 5: 920-926.

Skerk V, Pintarić H, Delić-Brkljacić D, Popović Z, et al. (2012). Orthostatic intolerance: postural orthostatic tachycardia syndrome with overlapping vasovagal syncope. Acta Clin. Croat. 51: 93-95.

Yao Y, Shi R, Wong T, Zheng L, et al. (2012). Endocardial autonomic denervation of the left atrium to treat vasovagal syncope: an early experience in humans. Circ. Arrhythm. Electrophysiol. 5: 279-286. 